\title{
A BIM-based supply chain model for AEC
}

\author{
E. Papadonikolaki, R. Vrijhoef \& J. W. F. Wamelink \\ Faculty of Architecture and the Built Environment, \\ Department of Real Estate and Housing, \\ Delft University of Technology, The Netherlands
}

\begin{abstract}
This paper proposes a model to integrate the construction Supply Chain (SC) through Building Information Modelling (BIM). The various information flows in the construction SC are vague. BIM is an aspiring integrator of information that could potentially improve such multi-disciplinary information flows. The paper presents a method to bridge information gaps and integrate the team using BIMbased SC modelling. After a literature review, the modelling method is presented. The model merges product and process models to social networks in a graph-based framework to represent a BIM-enabled SC. Although, BIM-enabled SC Management (SCM) is not widely applied, there is increasing interest from many stakeholders to engage in both BIM and SCM. The paper uses a real-world case as a proof of concept. The model analyses the actors' interactions, identifies bottlenecks and increases the collective understanding about the project.

Keywords: supply chain (SC), building information modelling (BIM), modelling and simulation (M\&S), graph-based model, case study research.
\end{abstract}

\section{Introduction}

Currently the Architecture Engineering and Construction (AEC) industry is complex due to the fragmented delivery processes, the building challenges and the large number of project stakeholders [1]. Building Information Modelling (BIM) is able to counterbalance such organisational, operational and technical complexities in the AEC Supply Chain (SC). Simultaneously, SC Management (SCM) has already proven effective in managing the material, information, cash and work crew flows among a set of strategically aligned companies. SCM establishes long-term trusting relations among the various SC partners by 
rendering mutual profits (London [2], Vrijhoef and Koskela [3]). Hence, BIM technology and SCM theory are alone both quite beneficial for the AEC.

$\mathrm{BIM}$ is a technology-driven approach that supports the AEC, by collecting and representing building project information and managing the information flows. For Eastman et al. [4], BIM is an integrative technology with "parametric intelligence". Among its accredited benefits, there are technical benefits, such as visualisation, automatic generation of drawings, code reviews, construction sequencing as well as operational benefits, such as cost and time reduction, negative risk reduction and scope clarification (Azhar [5], Liu et al. [6], Barlish and Sullivan [7], Bryde et al. [8]). Therefore, BIM undoubtedly reduces the technical and operational challenges of the construction industry.

However, the impact of BIM on the organisational challenges of the AEC SC is not thoroughly studied. BIM adoption and SCM approaches are only conceptually but not substantially combined in research. While Arayici et al. [9] and Sebastian [10] report on the changing dynamics in the roles of the stakeholders induced by BIM, its adoption within extended SC project teams, is not sufficiently investigated. Moreover, nowadays the project risks are indistinguishable due to multiple interactions among the SC actors [11]. This study researches the BIMenabled interactions among inter-organisational $\mathrm{SC}$ teams and proposes a sociotechnical approach for integrating BIM and SCM.

This paper presents a dynamic SC model using BIM as an integrator of information. It merges the technical, operational and organisational aspects of the AEC SC by combining BIM technology and SCM theory. Plus, it analyses the complexities of the products, operations and collaboration in a real-world case study. The rest of the paper contains the background, methodology and results from the test case study along with discussion and conclusion.

\section{Background}

\subsection{BIM technology for SCM research in AEC}

BIM technology has been linked to a variety of management approaches by exploring its built-in features in the software applications - such as Autodesk Revit, Bentley Architecture, Graphisoft Archicad, Nemetschek Allplan and many others - or by developing state of the art solutions. At the same time, SCM is successful only if the individual project management approaches of all the stakeholders are fruitful and not necessarily vice versa. Therefore, the internal managerial activities of an organisation are very crucial for the success of SCM.

Supply Chain thinking emerged from the areas of Operations Research (OR) and logistics, approximately in the mid-80s (London and Kenley [12]). Christopher [13] considered the SC a set of flows: downstream flow of material, upstream flow of transactions and bidirectional flow of information. Later, he [14] regarded a $\mathrm{SC}$ as a network and not a chain, since the multiple organisations that form it, generate different and multiple information streams simultaneously. Thus, a $\mathrm{SC}$ is a complex and distributed network of many organisations. 
Therefore, apart from the - already researched - flows of material, cash and information, the network of actors or stakeholders is additionally crucial for SCM. This literature review analyses BIM through these four areas of SC flows:

- Material flow;

- Cash flow;

- Information flow and

- Stakeholder network.

\subsection{BIM in support of the material and cash flows}

BIM supports the material flows in construction by reinforcing the existing and establishing new communication protocols and feedback mechanisms between the design and construction teams. For example, BIM ensures accurate feedback channels in remote construction projects [15] or monitors the supply chains by integrating tracking technologies to enhance the visibility during the material delivery $[16,17]$. Ergo, BIM is a multi-faceted integrator of processes for the monitoring and control of materials in the SC.

Likewise, BIM is used for calculating the cash flows and strengthening the feasibility and control of project cost analyses. The project benefits of BIM include cost reduction, enhanced control and time savings according to Bryde et al. [8]. Forgues et al. [18] claim that the cost estimating processes have changed enormously after the introduction of BIM. However, the BIM-based cost estimating processes do not completely replace the traditional methods as Hartmann et al. [19] point out. Thus, BIM apart from being a structured tool for controlling the material flows also sufficiently manages the SC cash flows.

\subsection{BIM in support of the information flows and the actors' network}

By definition, BIM facilitates and regulates the information flows. It offers methods for product modelling, interoperability and distribution of project information among the extended SC team. BIM ensures standardised product information with the Industry Foundation Class (IFC) model. Likewise, it offers collaboration via online platforms and improves the traditional data management. While the centrality of the BIM models is questionable, Van Berlo et al. [20] propose workarounds that offer decent BIM interoperability. Hence, the information flow, a core aspect of SCM, is sufficiently supported by BIM.

Sebastian [10] claims that BIM adoption causes changes not only in the products and processes, but also in the roles of contractors, architects and clients. The project team has been enlarged and parties that used to be supportive now play a dominant part in design and construction, such as property developers and facility managers. BIM adoption affects all the SC actors and it offers a potential for connecting the information flows throughout the project life cycle. Still, the exact impact of BIM and the new roles are not defined yet and the supply chains adopt new technologies without making the necessary managerial shift. 


\subsection{Gaps in the use of BIM for SCM}

Undoubtedly, the construction SC faces many challenges due to its unique main product. The AEC SC is a loosely interrelated system, and thus unreliable and inefficient, although it is central to the capital market and national economies. Azambuja and O'Brien [21] claim that the lack of sharing accurate, controllable and integral information among those organisations is responsible for this fragmentation of the industry. Hence, regulating the information flows by evaluating the options offered by BIM is essential for construction industry.

All the aforementioned benefits from BIM adoption in several SCM flows, suggest a positive impact from BIM-enabled SCM. However, there are yet unresolved issues, e.g. the actual BIM collaboration process among all partners and the establishment of a consistent information flow. Another problematic area is the large number of involved stakeholders, which should be managed in a structured manner. In the next sections, the paper will tackle these practical issues by proposing a modelling framework for the integration of BIM and SCM.

\section{Methodology}

\subsection{Starting point}

This study investigates the organisational, operational and technical facets of the AEC SC using Modelling \& Simulation (M\&S). M\&S was chosen as a natural methodology to integrate SCM and BIM. On one hand, modelling is among the main approaches for quantitative SC research as Seuring et al. [22] note. Simulation is the executable version of the model that provides dynamic insights in its operation. On the other hand, the native file format of BIM, the IFC, is a standard model of product information for AEC. BIM was initially introduced as Building Product Model (BPM) and it is basically a product model. Ergo, M\&S is a suitable approach for combining SCM and BIM.

This paper proposes a multi-model system for combining SCM theory and BIM technology. The multi-model approach responds to the system complexity by assimilating many facets (products, processes and people). After all, Papadonikolaki and Verbraeck [23] have previously underlined that the degree of modelling complexity should be proportional to the degree of system complexity. Thus, since a SC is a distributed system, could be also represented by a composition of modelling approaches. This rationale is explained next.

\subsubsection{Process and product modelling}

Process modelling represents a roadmap of activities within a firm or a complex system, e.g. the construction SC. This roadmap has a work breakdown structure and order plan. Such models are either static or dynamic. Static models represent only the structure of the system. The dynamic models represent the transformation of the system during time. State machines, stock and flow diagrams, activity diagrams and event graphs represent processes dynamically. Still, there is no 
formal methodology for the simulation of a SC and it mostly depends on the research domain and the chosen approach, as Reiner notes [24].

Product modelling refers to data specifications about a given artefact (physical or conceptual). The area of data modelling was developed to formalise these data logically (Eastman [25]). Dado et al. [26] state that the need to achieve a highlevel definition of the building systems, generated the area of product modelling by using the advancements in data modelling. The IFC model is a construction industry standard with definitions of mainly products, used for data modelling and interoperability in many proprietary applications. But, since it is an EntityRelation model, it lacks time-awareness and it faces a "process and data dependency problem" as Eastman [25] describes. Hence, the process and products models alone do not correspond to all the various complexities of a distributed and multi-faceted system, such as the AEC SC.

\subsubsection{Social networks}

The use of Social Networks is proposed to overcome these limitations of process and product modelling. The actors increase the complexity of the system at both intra- and inter-organisational level. Kornelius and Wamelink [27] suggested that the interweaving relationships in such networks cannot be represented in a bilateral basis. A Social Network (SN) composed of nodes (actors or stakeholders) represents the interactions of the SC partners via information flows (lines, arrows or edges). In this study, the organisation is considered the Unit of Analysis (UoA). The SN models are based on Network Theory, (a subset of Graph Theory) and offer visualisation. Pryke [28] performs SN analysis to visualise and explain the information exchanges and the contractual relationships in construction. Therefore, the SN models are capable to describe and analyse the organisational and social complexities of the construction SC.

\subsection{Implementation}

\subsubsection{Graphical representation}

Graphs are flexible modelling tools that consist of nodes and connecting lines. Graphs are popular in systems theory, software engineering and computer science. The basic module of a graph is a triple that consists of two nodes (or vertices) that are connected through an edge (line or arrow). Graphs are used in BIM research, to represent the hierarchical data models, i.e. IFC. Also, graph theory is suitable to exemplify SC research concepts, e.g. in the illustrations of O'Brien et al. [29] and London [2]. Thus, this graph-based approach can represent a complex system, such as the AEC SC, both visually and analytically.

The proposed model is the synthesis between process and product models and dynamic SN (UoA is the organisation) that combines BIM technology and SCM theory in AEC in a multi-model graph. The building block of the proposed model is shown in Figure 1. On top, it shows a fragment of the product model (the occurrence entities of IFC) shown as "product nodes" of an object hierarchy chart (white nodes). This product model gives information only about the content and the structure of information, neglecting time sequence or social interaction. In the middle, the "process node" (green node), connects with the product model, i.e. 
IFC. At the lower part of Figure 1, the "process node" connects with the social network or "actor nodes" (blue nodes). The arrows that join products to processes represent the input and output $(\mathrm{I} / \mathrm{O})$. The arrows that join actors to processes represent their interactions and are demarcated with roles inspired by Transaction Theory. The outcome is a multi-model synthesis of the three models: the product, process and SN model of the SC.
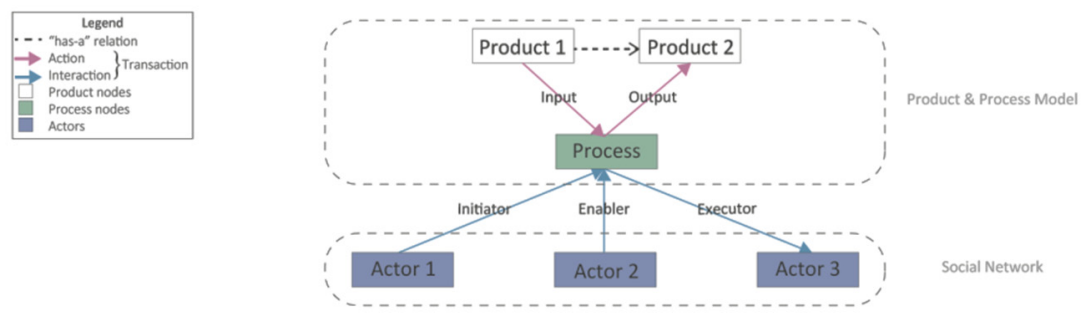

Figure 1: The building block of the graph-based model.

\subsubsection{Tabular representation and analysis}

The proposed model accumulates data from three sources: product, process and social models. First, it uses data from the product model (i.e. BIM) in the form of IFC files. The IFC files produced from all project actors are analysed with IFC File Analyzer, developed by the National Institute of Standards and Technology (NIST) agency of the US Department of Commerce. The format of this analysis is spreadsheet files. Second, the model uses data from the process model of the project again in spreadsheets. Third, the model uses information about the involved project actors. These different three data sources represent the product-, process- and people-related information about a project, respectively, and are combined in one relational database. This database is the basis for visualising and analysing a construction project.

The graph-based multi-model framework for a BIM-enabled SC is dynamic and created by the GEPHI tool. GEPHI is an open-source interactive visualisation and exploration platform for networks and complex systems, dynamic and hierarchical graphs developed by Bastian et al. [30]. This tool has a both graphical and a database environment and enables data import and export through tabular files. The aforementioned relational database is imported as a set of spreadsheets. The spreadsheets are then combined in GEPHI by establishing their relations. Finally, the data are exported to R programming environment for further statistical risk analysis with $\mathrm{R}$ language, developed by RCoreTeam [31].

\subsubsection{Model design requirements}

The model uses BIM and SCM theory to propose a framework for SC integration. The simulation analyses the system and produces an array of dynamic insights. To guarantee its usability and usefulness, the theoretical input is combined with input from practice. Before applying the model to real-world test cases, five guiding model design requirements were drafted to ensure the adherence to the research 
goals. These following modelling requirements describe how to apply the model to real-world case studies as well as the desired output of this modelling study:

- The model should contain the information flows from BIM applications.

- The model should be applicable and extendable to different SC projects.

- The model when applied to a case should analyse the project phases.

- The model should produce quantitative results for further analysis.

- The model and its output should be acceptable by the SC actors.

\section{Data analysis and findings}

\subsection{Case description}

The scenario test case is an ongoing real-world case of a BIM-enabled SC. The project was selected because it is large scale and has a diverse team and various technical risks. It is new construction of a multi-functional complex of 255 apartments, offices and shops, divided in 3 buildings. It has special energy requirements (incorporation of renewable sources; geothermal and solar). Since it is still ongoing, only the initial phases are simulated with the proposed model.

The project is located in Utrecht, Netherlands and consists of ten SC partners: client, contractor, architect, structural engineer, facility manager, energy adviser and four engineering and installation firms (HVAC). The architect, client and heating firm are new and external to the SC. The rest have worked together in three other projects, one BIM-enabled. The project SC is well defined but not yet integrated. They have a "no-blame" culture during their daily interaction. They have issued their own BIM protocol and hold fortnightly BIM meetings for collaboration and informal weekly BIM sessions for training.

\subsection{Case analysis}

The modelled overview of the case is shown in Figure 2. It contains all the actions and interactions among the modelling entities and illustrates the project complexity. The product model is the list of IFC entities on the top. The process model is an array of green nodes in the middle and the actors are the set of blue nodes below. The links - or relations - among the different models, are shown with arrows. The links between the product and the process nodes are input, means or output (pink colour). The relations between the process and actor nodes have notations from Transaction Theory, e.g. initiator, enabler and executor (blue colour). Since the case is ongoing, the last phases are not yet modelled.

\subsection{Results}

Apart from offering a structured model for representing the technical, operational and organisational complexities of a BIM-enabled SC, this model offers a set of dynamic insights into the SC. Figure 3 contains six analyses of the modelled test case. Since the project is still ongoing, the estimations for the remaining phases (from preparation to operation) appear as dashed lines in the diagrams of Figure 3. 


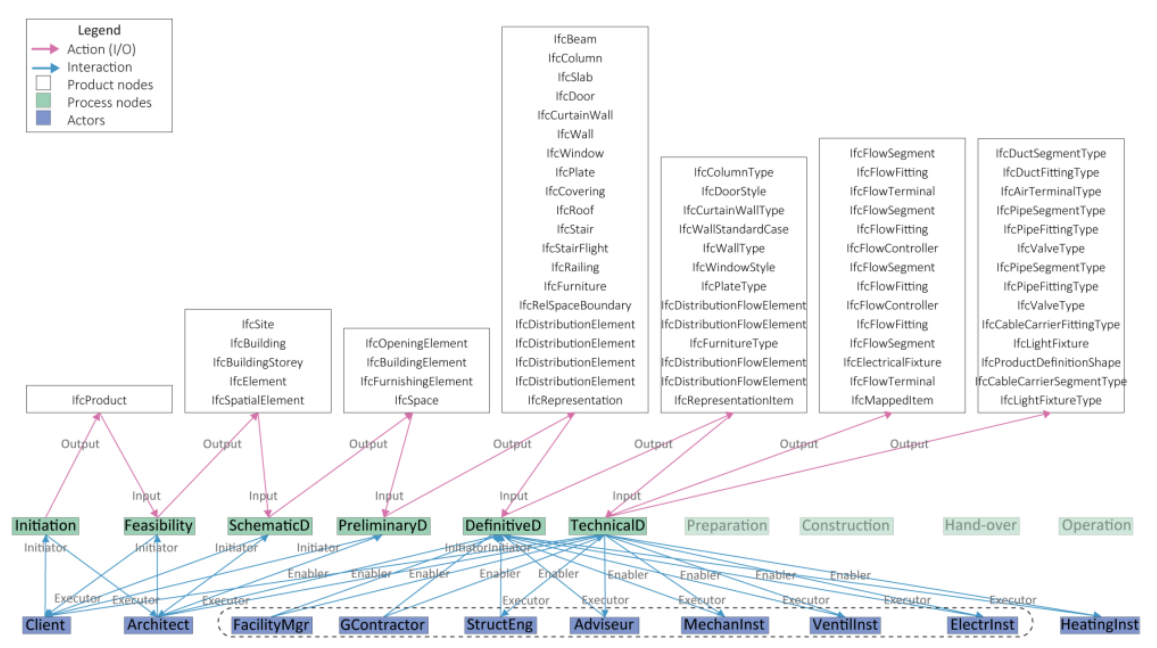

Figure 2: Overall view of the model for the ongoing test case.

The following quantitative diagrams illustrate the project complexity in terms of technical (a) and (b), operational (c) and (d) and organisational (e) and (f) complexity. The model has 92 modelling entities: 10 actors, 10 phases and 72 product entities (Figure 3(a)). The technical complexity emerges from the Preliminary Design phase when all co-makers input their entities in the BIM model (Figure 3 (b)). While the first phases last quite long, the Definitive Design up to Construction have pressing deadlines (Figure 3(c)). This becomes more intense since the interactions among the SC actors also increase greatly then (Figure 3 (d)). Regarding the organisational complexity, the various SC actors are active in different degrees and thus their interaction is complex and fragmented (Figure $3(\mathrm{e})$ ). The roles of each SC actor are grouped as active (initiator and executor) or passive (enabler) (Figure 3(f)).

\section{Discussion}

\subsection{Social impact}

The proposed model analyses a BIM-enabled SC, focusing on interactions and roles. The model combines BIM technology and SCM theory and integrates the products, processes and people. The model conformed to the previously set requirements (section 3.2.3) and performed the following actions in the test case:

- Integration of product data from BIM (IFC) to structure the information.

- Scalability of the model according to various tasks and projects.

- Monitoring of the SC project phases, the requirements $(\mathrm{I} / \mathrm{O})$ and roles.

- Quantifiable analyses of the SC project in tables and diagrams.

- Pending dissemination and validation since the project is still ongoing. 


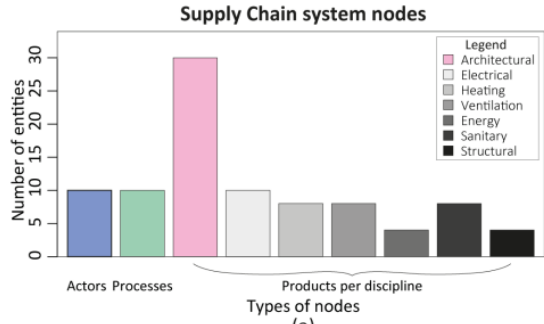

(a)

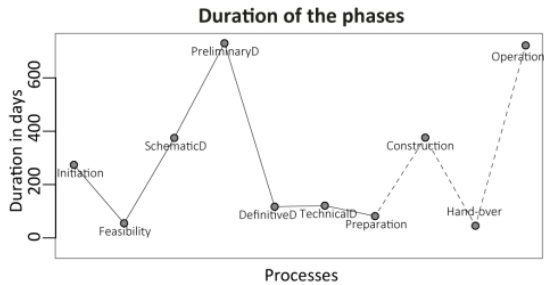

(c)

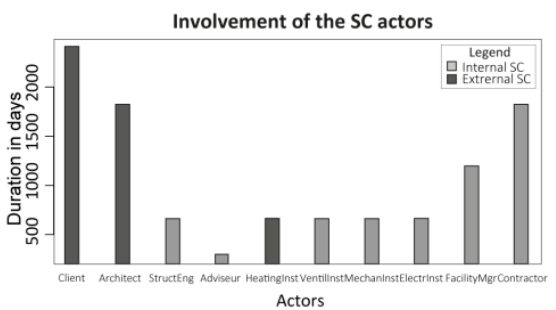

(e)

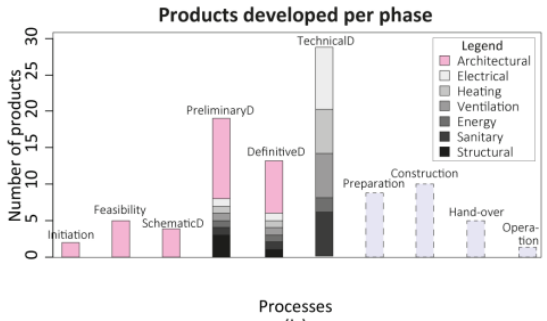

(b)

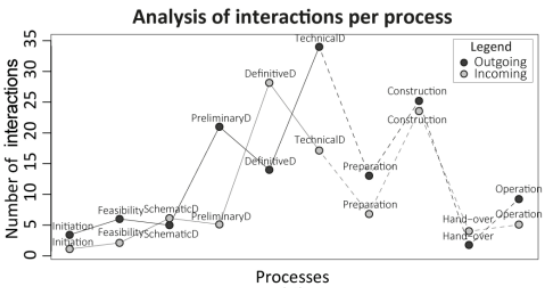

(d)

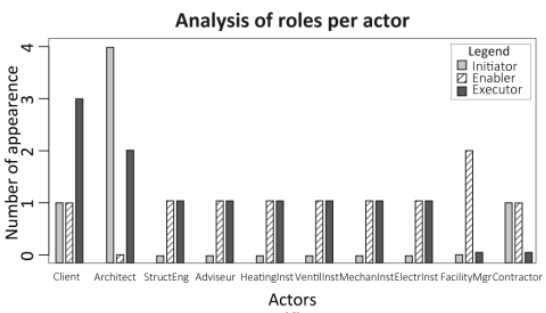

(f)

Figure 3: Diagrams extracted from the model for analysis of the product (a) and (b), process (c) and (d) and organisational (e) and (f) complexity respectively.

The BIM-enabled collaboration lacks trust as Miettinen and Paavola [32] and Cao et al. [33] claim. Mutual trust and enhanced commitment to the SC are also primary goals of SCM. The narratives from the test case confirm that the BIMenabled collaboration was smooth even though the SC composition was not uniform. In particular, the architect and client played central roles to the project (Figure 3(e) and (f)) even though they were external to the SC. Plus, the model analysis confirms Shi [11] that the actors' interactions increase greatly even though the time pressure increases (Figure 3(c) and (d)).

The SC stakeholders interact in a complex manner. The existing BIM-based collaboration tools only emphasise on access to information, but do not support an analysis of the actors' interactions. These unclear and unregulated interactions among the actors result to poor information management. With this model, SCM theory supports a method to enrich BIM practice by emphasising on the interactions and roles and inspiring mutual commitment and responsibility. 


\subsection{Technical considerations}

Although the IFC is used in BIM applications as a standard product model, the need for even greater standardisation is evident. Ontology-based knowledge structures re-emerge, due to the standardisation of the Web Ontology Language (OWL) in 2009. Semantic Web applications are used to improve the redundancy of the IFC, the complexity of the semantics, to compensate for the information loss (Pauwels et al. [34]) and to enhance interoperability (Venugopal et al. [35]). In the proposed graph-structured model, the information is organised in RDF triples (Resource Description Framework) for future semantic web integration, taking the relevant state of the art in research into account (see again Figure 1).

The model collects product information from BIM via the IFC. However, the IFC has often being accused of losing the reliability and integrity of information. In the proposed model, the occurrence entities of IFC are used for providing the structure of building information and not for detailed geometric, material or energy information. Currently, the model uses a rudimentary analysis of the IFCs performed by the NIST IFC File Analyzer. In the future, a more sophisticated tool or a specialised filtering algorithm will be implemented.

\section{Conclusion}

The adoption of BIM is fragmented and applied in an ad-hoc manner, despite its acknowledged benefits and the advancements in computing infrastructure. Likewise, although SCM in construction becomes a hot-topic after recognising its financial returns, the AEC SC is inefficient, unreliable and fragmented. The proposed multi-model framework for SC integration identifies and analyses the organisational, operational and technical complexities and utilises the full potential of BIM technology and SCM theory, by using BIM as an information integrator and SCM as a trusting collaboration environment respectively.

Building product and process data (to represent the state and time changes respectively) combined with stakeholders' information (to represent the interactions among the SC partners) are joined to a structured representation and analysis framework for the AEC SC. In a real-world case study, the model performed dynamic risk analyses and offered an overview of the SC collaboration. This BIM-based SC model includes an analysis of the building information flows from each actor's BIM model and an analysis of the project phases. Additionally, it includes soft parameters and identifies the projects risks focusing on the interactions and roles of the participants. In the future, the model will be applied to additional real-world cases for calibration in order to establish a promising framework for the integration of BIM-enabled SC partnerships.

\section{References}

[1] Zavadskas, E.K., Z. Turskis, and J. Tamošaitiene, Risk assessment of construction projects. Journal of civil engineering and management, 16(1): pp. 33-46, 2010. 
[2] London, K., Industrial Organization Object-Oriented Project Model of the Facade Supply Chain Cluster, in Construction Supply Chain Management Handbook, W.J. O'Brien, et al., Editors, CRC Press: Boca Raton, Florida, USA, p. 13-1-13-46, 2009.

[3] Vrijhoef, R. and L. Koskela, The four roles of supply chain management in construction. European Journal of Purchasing and Supply Management, 6(3-4): pp. 169-178, 2000.

[4] Eastman, C., et al., BIM Handbook: A Guide to Building Information Modeling for Owners, Managers, Designers, Engineers, and Contractors. Second ed. Hoboken, New Jersey, USA: John Wiley \& Sons Inc., 2008.

[5] Azhar, S., Building Information Modeling (BIM): Trends, Benefits, Risks, and Challenges for the AEC Industry. Leadership and Management in Engineering, 11(3): pp. 241-252, 2011.

[6] Liu, R., R. Issa, and S. Olbina, Factors influencing the adoption of building information modeling in the AEC Industry, in Proceedings of the International Conference on Computing in Civil and Building Engineering (ICCCBE 2010), Nottingham, United Kingdom, pp. 139-145, 2010.

[7] Barlish, K. and K. Sullivan, How to measure the benefits of BIM - A case study approach. Automation in Construction, 24: pp. 149-159, 2012.

[8] Bryde, D., M. Broquetas, and J.M. Volm, The project benefits of Building Information Modelling (BIM). International Journal of Project Management, 31(7): pp. 971-980, 2013.

[9] Arayici, Y.C.P.; Koskela, L.; Kagioglou, M.; Usher, C.; O’Reilly, K., BIM adoption and implementation for architectural practices. Structural Survey, 29(1): pp. 7-25, 2011.

[10] Sebastian, R., Changing roles of the clients, architects and contractors through BIM. Engineering, Construction and Architectural Management, 18(2): pp. 176-187, 2011.

[11] Shi, D., A review of enterprise supply chain risk management. Journal of systems science and systems engineering, 13(2): pp. 219-244, 2004.

[12] London, K. and R. Kenley, An industrial organization economic supply chain approach for the construction industry: A review. Construction Management and Economics, 19(8): pp. 777-788, 2001.

[13] Christopher, M., Logistics and supply chain management: Strategies for Reducing Cost and Improving Services. 2 ed. London, UK: Financial Times Professional Ltd., 1992.

[14] Christopher, M., Logistics and supply chain management: Creating ValueAdding Networks. 3 ed. New York, USA: Financial Times Prentice Hall, 2005.

[15] Arayici, Y., C. Egbu, and P. Coates, Building information modelling (Bim) implementation and remote construction projects: Issues, challenges, and critiques. Electronic Journal of Information Technology in Construction, 17: pp. 75-92, 2012.

[16] Chavada, R., N. Dawood, and M. Kassem, Construction workspace management: the development and application of a novel $\mathrm{nD}$ planning 
approach and tool. Journal of Information Technology in Construction, 17: p. 24, 2012.

[17] Irizarry, J., E.P. Karan, and F. Jalaei, Integrating BIM and GIS to improve the visual monitoring of construction supply chain management. Automation in Construction, 31: pp. 241-254, 2013.

[18] Forgues, D., et al., Rethinking the cost estimating process through 5D BIM: A case study, in Construction Research Congress 2012: Construction Challenges in a Flat World: West Lafayette, Indiana, USA. pp. 778-786, 2012.

[19] Hartmann, T., et al., Aligning building information model tools and construction management methods. Automation in construction, 22: pp. 605-613, 2012.

[20] Van Berlo, L., et al., Collaborative engineering with IFC: new insights and technology, in 9th European Conference on Product and Process Modelling, Iceland, 2012.

[21] Azambuja, M. and W.J. O’Brien, Construction Supply Chain Modeling: Issues and Perspectives, in Construction Supply Chain Management Handbook, W.J. O’Brien, et al., Editors, CRC Press: Boca Raton, Florida, USA. p. 2-1-2-31, 2009.

[22] Seuring, S., et al., Is There a Right Research Design for Your Supply Chain Study? in Research Methodologies in Supply Chain Management, H.S. Kotzab, Stefan; Müller, Martin; Reiner, Gerald, Editor, Physica-Verlag: Heidelberg, Germany. pp. 1-12, 2005.

[23] Papadonikolaki, E. and A. Verbraeck, Modelling and simulation research for construction supply chains, in eWork and eBusiness in Architecture, Engineering and Construction, CRC Press. pp. 615-622, 2014.

[24] Reiner, G., Supply Chain Management Research Methodology Using Quantitative Models Based on Empirical Data, in Research Methodologies in Supply Chain Management, H. Kotzab, et al., Editors, Physica-Verlag: Heidelberg, Germany. pp. 431-444, 2005.

[25] Eastman, C.M., Building Product Models: Computer Environments, Supporting Design and Construction. Boca Raton, Florida, USA: CRC Press, 1999.

[26] Dado, E., R. Beheshti, and M. van de Ruitenbeek, Product modelling in the building and construction industry: a history and perspectives. Handbook of Research on Building Information Modelling and Construction Informatics: Concepts and Technologies, Hershey, PA, IGI Global Publishing: pp. 104137, 2010.

[27] Kornelius, L. and J. Wamelink, The virtual corporation: learning from construction. Supply Chain Management: An International Journal, 3(4): pp. 193-202, 1998.

[28] Pryke, S., Towards a social network theory of project governance. Construction Management and Economics, 23(9): pp. 927-939, 2005.

[29] O'Brien, W.J., K. London, and R. Vrijhoef, Construction supply chain modeling: a research review and interdisciplinary research agenda, in 10th 
Conference of the International Group for Lean Construction: Gramado, Brazil. pp. 64-84, 2002.

[30] Bastian, M., S. Heymann, and M. Jacomy, Gephi: an open source software for exploring and manipulating networks, in International AAAI Conference on Weblogs and Social Media (ICWSM 8): San Jose, California, USA. pp. 361-362, 2009.

[31] RCoreTeam, R: A language and environment for statistical computing, in $\mathrm{R}$ Foundation for Statistical Computing: Vienna, Austria, 2013.

[32] Miettinen, R. and S. Paavola, Beyond the BIM utopia: Approaches to the development and implementation of building information modeling. Automation in construction, 43: pp. 84-91, 2014.

[33] Cao, D., et al., Practices and effectiveness of building information modelling in construction projects in China. Automation in Construction, 49: pp. 113-122, 2015.

[34] Pauwels, P., et al., Industry Foundation Classes: A Space-Based Model Scheme? in 26th eCAADe Conference Proceedings: Antwerpen, Belgium. pp. 117-124, 2008.

[35] Venugopal, M., et al., Semantics of model views for information exchanges using the industry foundation class schema. Advanced Engineering Informatics, 26(2): pp. 411-428, 2012. 